\title{
Mandibular distraction osteogenesis in the treatment of pediatric temporomandibular joint ankylosis with micrognathia and obstructive sleep apnea syndrome: A case report with 4-year follow-up
}

\author{
XINGNA YU*, JIANNING WANG*, SHIDA HOU and RONGSHENG ZENG \\ Department of Oral and Maxillofacial Surgery, Guanghua School of Stomatology, Hospital of Stomatology, \\ Sun Yat-sen University, Guangzhou, Guangdong 510055, P.R. China
}

Received March 29, 2019; Accepted October 2, 2019

DOI: $10.3892 /$ etm.2019.8119

\begin{abstract}
Temporomandibular joint (TMJ) ankylosis in pediatric patients is rare and may cause severe micrognathia and obstructive sleep apnea syndrome. The present study reports on the treatment and 4-year follow-up of a pediatric patient with early-onset bilateral TMJ ankylosis and severe secondary micrognathia, as well as obstructive sleep apnea syndrome. A typical 'bird face' appearance was noted with severe mandible retrognathism and a significant convex facial profile. The treatment of this patient involved TMJ ankylosis release with condylectomy and simultaneous bilateral mandibular distraction osteogenesis, which enabled the surgeons to simultaneously reconstruct the neocondyle and correct facial malformations. Following treatment, the micrognathia was corrected and the oropharyngeal airway was significantly expanded. However, the maximal incisal opening was limited. During the 4-year follow-up, no signs of mandible retraction were noted and mouth opening increased to $17 \mathrm{~mm}$ (passive) compared with the inability to open that was noted immediately following surgery. A certain degree of MIO shrinkage was identified in the patient. In such cases of TMJ ankylosis, early post-operative exercise, active post-operative
\end{abstract}

Correspondence to: Dr Rongsheng Zeng, Department of Oral and Maxillofacial Surgery, Guanghua School of Stomatology, Hospital of Stomatology, Sun Yat-sen University, 56 Lingyuan West Road, Guangzhou, Guangdong 510055, P.R. China

E-mail: rszengjoms@163.com

*Contributed equally

Abbreviations: TMJ, temporomandibular joint; OSAS, obstructive sleep apnea syndrome; MDO, mandibular distraction osteogenesis; MIO, maximal incisal opening

Key words: temporomandibular joint ankylosis, mandibular distraction osteogenesis, micrognathia, obstructive sleep apnea syndrome physiotherapy and stringent follow-up are essential to prevent post-operative shrinkage and adhesions.

\section{Introduction}

Temporomandibular joint (TMJ) ankylosis refers to immobility of the joint and is characterized by the formation of an osseous, fibrous, or fibro-osseous mass fused to the cranial base (1). A typical 'bird face' appearance is noted in cases of bilateral ankylosis with onset during childhood (2). Restriction of mandible movement in pediatric patients frequently leads to physical and psychological impairments, including speech impairment, difficulty in chewing and swallowing, rampant caries, poor oral hygiene, facial deformity and airway obstruction. Furthermore, the condition of micrognathia is thought to cause obstructive sleep apnea syndrome (OSAS) due to airway obstruction due to glossoptosis and reduced oropharyngeal space (3).

Several surgical methods have been applied to treat patients with micrognathia and repair their bone defects. However, mandibular distraction osteogenesis (MDO) has additional advantages over other methods (4). MDO is able to correct the micrognathia and relieve the airway obstruction due to its ability to stretch the tongue and muscles attached to the mandible forward (5). The present study reports on a case of micrognathia and OSAS due to bilateral TMJ ankylosis in early childhood. TMJ management combined with simultaneous MDO was used for the treatment of this patient and the 4-year follow-up indicated optimal results.

\section{Case report}

Case presentation. A 5-year-old male patient presented with an abnormal mandible, limited mouth opening and snoring. The cause of the TMJ ankylosis was condylar fracture resulting from a trauma to the chin when the patient was 2 years old. The TMJ exhibited no condylar mobility and the intraoral condition was poor with several teeth affected by caries and residual roots. A typical 'bird face' appearance with serious mandible retrognathism and significant convex facial profile was noted (Fig. 1). In addition, the patient was unable to lie flat on the bed at night, resulting in pronounced snoring. Due to poor cooperation of 
the patient to perform the polysomnography test and economic considerations, no oxygen saturation or apnea/hypopnea index data were collected. However, CT measurement was used for pharyngeal space measurement instead of MRI estimation. On CT, the pharyngeal space was narrow with a width of $13.08 \mathrm{~mm}$ and a length of $2.12 \mathrm{~mm}$ in the retroglossal region (Fig. 2). Radiological examinations confirmed a condyle deformity and the mandible was apparently underdeveloped downward and forward. Cephalometric analysis indicated a severe skeletal Class II malocclusion with an angle that reflects the maxillary protrusion, formed by the sella turcica point, nasion point and upper alveolar point (SNA), of $79.5^{\circ}$; an angle that reflects the mandibular protrusion, formed by sella turcica point, nasion point and inferior alveolar point (SNB), of $60.1^{\circ}$; and an angle that reflects the relative protrusion of maxilla and mandible, formed by the upper alveolar point, nasion point and inferior point (ANB) of $19.4^{\circ}$. A severely hypoplastic mandible and chin with a facial angle of $67.8^{\circ}$ and a Y-axis angle of $73.1^{\circ}$ were also observed (Table I). The diagnosis was TMJ ankylosis, micrognathia and OSAS. A cephalometric map used for the measurements is provided in Fig. 3. Calculation of the pharynx diameters was accomplished by using the software Mimics Research version 20.0 (Materialise Corp.). The same slice was identified by the lower border of the second cervical centrum, which was the middle part of the oropharynx and the narrowest point of the pharyngeal airway in this patient. Repeated evaluations were performed for a total of three or four times for each measure to obtain the average value and statistical analysis was not applicable to this study.

Treatment procedure. The protocol by Kaban et al (6) was followed during the initial surgery. The first step was TMJ arthroplasty by condylectomy and simultaneous MDO, with bilateral coronoidectomy if required. During the procedure, the surgeons concluded that the normal TMJ architecture and intact disc had not been retained and that the ankylotic mass was fused to the skull base. Subsequently, a specimen of $15 \mathrm{~mm}$ in length of the ankylosed condyle was excised at the level below the condyle neck. The maximal opening measured $35 \mathrm{~mm}$ after completion of the excision. To avoid recurrence of ankylosis, a Dacron patch (Chester Healthcare Technology Co., Ltd) was sutured to the soft tissue. The use of a temporalis muscle fascia flap was avoided following condylectomy.

An individualized 3D-printed model (Cibei Medical Treatment Appliance Co., Ltd) was constructed prior to the surgery and the osteotomy line was accurately designed on the mandible model in order not to injure the teeth buds. The osteotomy line was designed on the mandible angle to create a transport disc so that distractors were able to vertically lengthen the ramus height and sagittal plane, leading to a concomitant extension of the mandibular body. Cutting guides were also designed to fit on the mandible model in order to reproduce the planned osteotomy. Therefore, the parallel placement of the distraction devices was ensured by the same slots, which were designed on the cutting guides and devices. During the surgery, the corresponding screw holes were initially drilled on the bone in order to determine the positions of the distractors. Subsequently, the corticotomy was achieved in the mandibular angle. Finally, the internal distractors (Cibei Medical Treatment Appliance Co., Ltd) were fixed to the transport disc and mandibular body with screws, lengthening the ramus height and mandibular body.

After a latency period of 3 days, the distractors were activated three times daily by a $0.4-\mathrm{mm}$ turn. The duration of distraction lasted for 13 days until the anterior overjet disappeared and the anterior teeth exhibited a 2-mm crossbite. The total extracted length was $15.6 \mathrm{~mm}$. Physical training was initiated from the day when distractors were applied and a T-shaped opener was provided to the patient in order to aid with the training of the mouth opening. The patient's snoring symptoms were relieved immediately following surgery. Airway enlargement was evident on $\mathrm{CT}$ scans after the distraction period (Fig. 2D). The transverse view of CT scans indicated that the pharynx diameters were increased to $23.95 \mathrm{~mm}$ (width) and $7.70 \mathrm{~mm}$ (length). These were considerably greater than those determined prior to the operation $(13.08 \times 2.12 \mathrm{~mm})$.

Treatment and outcomes at the 1-year follow-up. No complications of toothache, wound infection or loosing of devices were reported. During removal at 1 year, it was noted that the distractors were still fixed in a good position and the screws were stable. According to the surgery record, the actual regeneration length was $15.5 \mathrm{~mm}$. The patient reported significant improvement with sleep problems as a result of the increase in the oropharyngeal airway. The distractors were removed following the consolidation period. The 'bird face' appearance was improved (Fig. 1B-E). Cephalometric measurements demonstrated that the SNB was increased to $67.0^{\circ}$, the ANB was decreased to $13.1^{\circ}$, the facial angle was increased to $73.6^{\circ}$ and the Y-axis angle was decreased to $70.4^{\circ}$. CT scans indicated further enlargement of the upper airway diameters that were estimated at $23.95 \mathrm{~mm}$ in width and $7.70 \mathrm{~mm}$ in length (Fig. 2F). However, the maximal incisal opening (MIO) returned to $11 \mathrm{~mm}$ at 1 year following condylectomy, since the patient was unwilling to endure the pain during mouth opening exercises. In order to obtain a larger opening capacity, a coronoidectomy was performed and the passive MIO reached to $30 \mathrm{~mm}$ following the completion of the surgery. Mouth-opening exercises were strongly recommended for the patient.

Outcomes at 4-year follow-up. The patient's lateral view improvement was basically maintained at 4 years following distraction (Fig. 1C). New articulation was eventually formed between the glenoid fossa and the transport segment following remodeling of this structure. The snoring symptom of the OSAS was resolved to a great extent. Cephalometric evaluation indicated significant improvements of the mandible atrophy (Fig. 1F) with an SNA of $80.3^{\circ}$, an SNB of $68.6^{\circ}$ and an ANB of $11.7^{\circ}$. The facial angle increased to $77.8^{\circ}$ and the Y-axis angle decreased to $69.9^{\circ}$. CT results indicated an enlarged pharynx space with a transverse diameter of $30.9 \mathrm{~mm}$ in width and $6.30 \mathrm{~mm}$ in length (Fig. 2H). However, the patient's MIO was estimated to $14 \mathrm{~mm}$ and $17 \mathrm{~mm}$ for the active and passive state, respectively. There was a certain degree of MIO shrinkage in the patient. The present MIO result did not reach the optimal expected outcome due to the lack of physical training and poor compliance. The patient had no regular follow-up schedule due to the long journey from his residency to the hospital. Therefore, further 
A

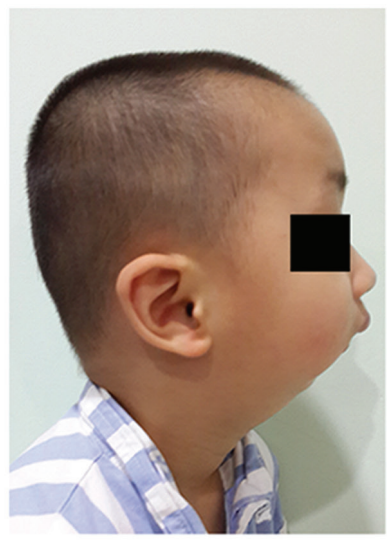

D

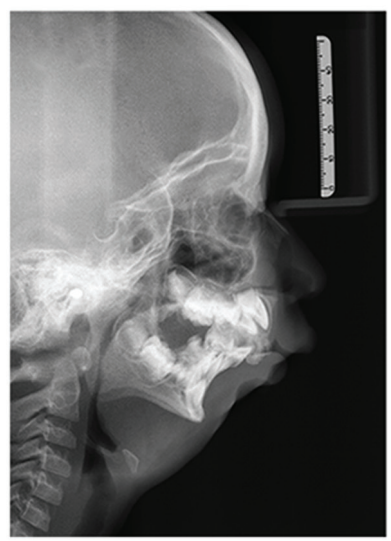

B

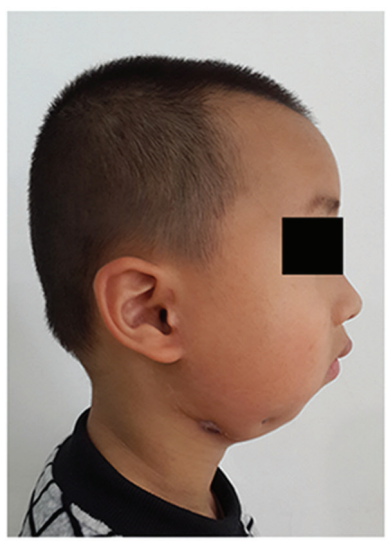

E

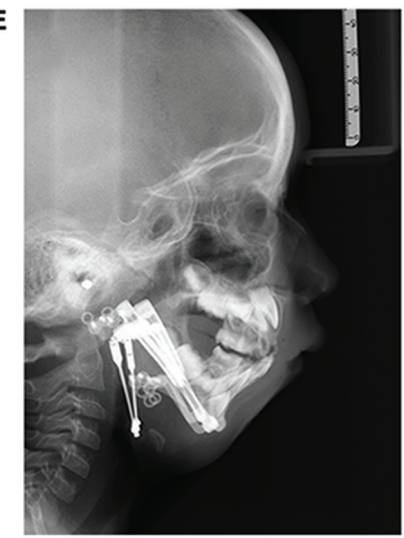

C
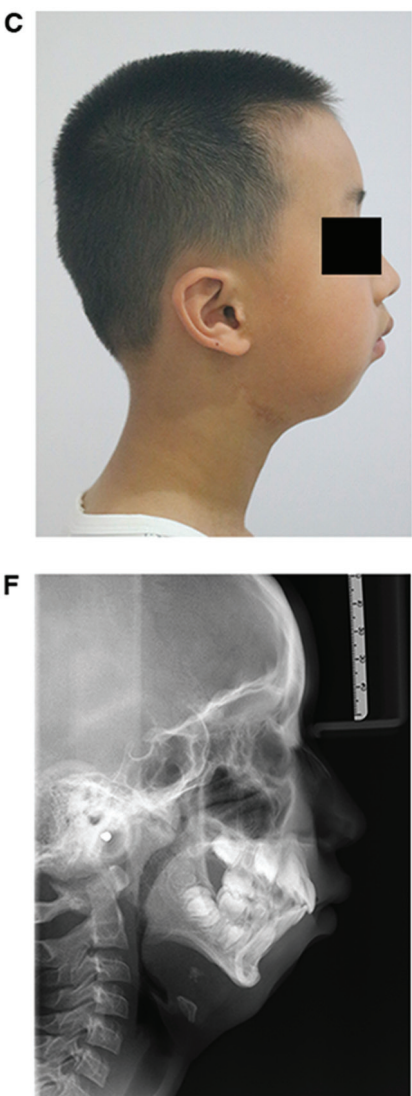

Figure 1. Lateral changes during the distraction process. Lateral images (A) prior to distraction, (B) post-distraction and (C) at 4-year follow-up. Lateral X-ray images (D) prior to distraction, (E) post-distraction and (F) at 4-year follow-up.

A
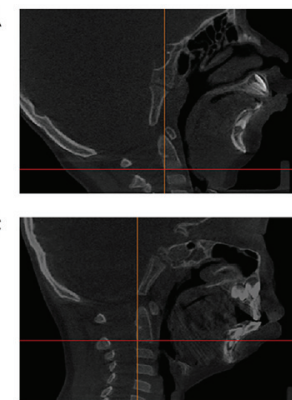

E
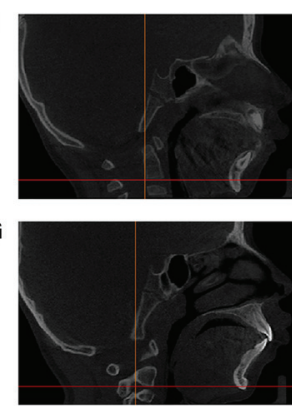
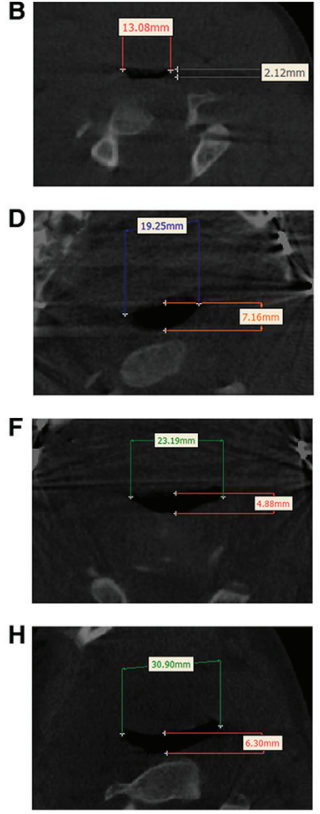

Figure 2. CT scans of oropharyngeal airway during the distraction process. CT sagittal and transversal views on the same slice level at different timepoints during distraction. CT scans (A) before distraction, (B) pre-distraction transverse airway diameters, which were narrow in the retroglossal region, (C) sagittal view immediately after distraction, (D) transverse diameters that were enlarged immediately following distractions, (E) sagittal view 8 months following distraction, $(F)$ transverse view showing airway enlargement at 8 months following distraction, indicating that the airway diameters were further enlarged, $(\mathrm{G})$ sagittal view at 4-year follow-up, and $(\mathrm{H})$ transverse view indicating that the airway diameters continued to broaden (specific measures provided in the images).

physiotherapy with good compliance were required for the patient.

\section{Discussion}

The present study describes a case of pediatric bilateral TMJ ankylosis and severe secondary micrognathia and OSAS. In this patient, TMJ ankylosis occurred at a young age as a result of mandibular trauma and caused severe facial deformity and airway obstruction. In addition to the growth, the patient was burdened with severe functional and aesthetic malformations that may adversely affect his social and psychological development (7). Therefore, it has been proposed that early treatment of ankylosis is necessary to avoid secondary deformities $(6,7)$. In the present case, condylectomy was performed at the age of 5. The patient was of sufficient age to cooperate with post-operative physiotherapy. However, the patient's MIO indicated limited improvement following treatment, due to poor compliance. Therefore, the study suggested that, although early surgical treatment is essential for pediatric patients with ankylosis, patient compliance, family support and the financial burden should also be considered during the selection of the appropriate time for surgical correction. Early post-operative exercise, active physiotherapy and stringent follow-up are essential to prevent post-operative shrinkage and adhesions in TMJ ankyloses. The motivation to perform active mouth opening training and tolerance of discomfort are considered key factors in determining successful maintenance of post-operative mouth opening (8). 
Table I. Cephalometric analysis throughout the distraction process.

\begin{tabular}{|c|c|c|c|c|}
\hline Measurement & $\begin{array}{l}\text { Pre-treatment: } \\
\text { Age, } 5 \text { years } \\
\text { and } 5 \text { months }\end{array}$ & $\begin{array}{l}3 \text { months after } \\
\text { mandibular DO: Age, } \\
5 \text { years and } 8 \text { months }\end{array}$ & $\begin{array}{l}\text { Post-treatment: } \\
\text { Age, } 9 \text { years } \\
\text { and } 4 \text { months }\end{array}$ & $\begin{array}{c}\text { Normal value } \\
\text { on mixed dentition }{ }^{\mathrm{a}}\end{array}$ \\
\hline \multicolumn{5}{|l|}{ Angle $\left({ }^{\circ}\right)$} \\
\hline SNA & 79.5 & 80.1 & 80.3 & $82.3 \pm 3.5$ \\
\hline SNB & 60.1 & 67.0 & 68.6 & $77.6 \pm 2.9$ \\
\hline ANB & 19.4 & 13.1 & 11.7 & $4.7 \pm 1.4$ \\
\hline Facial angle & 67.8 & 73.6 & 77.8 & $83.1 \pm 3.0$ \\
\hline Y-axis angle & 73.1 & 70.4 & 69.9 & $65.5 \pm 2.9$ \\
\hline Li-Pog'-throat angle & 175.6 & 161.7 & 159.6 & $110.0 \pm 8.0$ \\
\hline \multicolumn{5}{|l|}{ Linear distance (mm) } \\
\hline Co-Gn & 62.4 & 69.0 & 80.1 & - \\
\hline Me'-throat length & 24.4 & 27.3 & 30.8 & $42.0 \pm 6.0$ \\
\hline Chin projection & -26.9 & -21.0 & -17.4 & $0 \pm 2.0$ \\
\hline
\end{tabular}

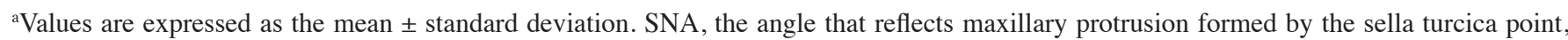
nasion point and upper alveolar point; SNB, the angle that reflects mandibular protrusion formed by sella turcica point, nasion point and inferior alveolar point; ANB, the angle that reflects the relative protrusion of maxilla and mandible formed by upper alveolar point, nasion point and inferior point; facial angle, the lower posterior angle of the intersection of N-Pog line and Frankfort horizontal plane; Y-axis angle, lower anterior angle of the intersection of the S-Gn line and Frankfort horizontal plane; Li-Pog'-throat angle, upper posterior angle of the intersection of the Li-Pog' line and throat line; Me'-throat length, distance from Me' to the throat angle; chin projection, vertical distance from Pog' to 0-degree meridian; y, years; mon, months. Me', submental point on soft tissue; Pog', pogonion on soft tissue.

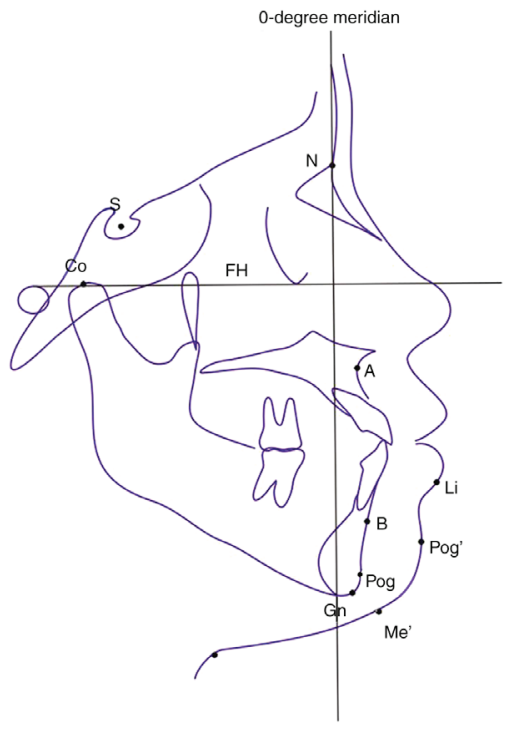

Figure 3. Cephalometric analysis. Landmarks of the hard tissue: S, sella turcica point; N, nasion point; A, upper alveolar point; B, inferior alveolar point; Pog, pogonion; Gn, gnathion; Co, condyle point. Landmarks of the soft tissues: Li, labium; Pog', pogonion of the soft tissue; $\mathrm{Me}^{\prime}$, menton of the soft tissue; FH, Frankfort horizontal plane; 0-degree meridian, a line passing through the nasion that is perpendicular to the FH plane.

The purpose of MDO in the present case study included the correction of craniofacial deformities and the resolution of OSAS. The mandibular lengthening obtained by gradual distraction may result in expansion of the mandibular bony tissue and in proportional and harmonic modification of the muscles and the surrounding soft tissues (9). However, whether distraction should be performed at the time of TMJ management remains controversial. Certain surgeons prefer to first restore the jaw movements and address the secondary facial deformities afterwards (10), whereas others perform the distraction first, followed by TMJ management at the second stage (11). The selection of different surgical methods is based on specific factors. Lopez and Dogliotti (10) suggested that the MDO should be performed following arthroplasty, as the growth potential of the mandible is only realized when the ankylosis has been relieved. However, Sadakah et al (11) recommended postponing the release of the ankylosed joint following bilateral distractions, since it is more advantageous in preventing rotation and upward movement of the condylar segment during the course of the distraction. In contrast to this type of treatment, it has been proposed by surgeons to attempt simultaneous correction of all deformities by performing distraction during ankylotic mass removal following the introduction of distraction osteogenesis for mandibular lengthening (12). Simultaneous arthroplasty and MDO enable the production of a neocondyle and allow for concomitant correction of facial malformations. The treatment outcomes of pre-arthroplastic distraction and simultaneous arthroplastic distraction have been previously compared and it was concluded that the two methods were effective in correcting the aesthetics of the patients and functional movement of the TMJ (13). Papageorge and Apostolidis (14) were the first to perform simultaneous gap arthroplasty and MDO for the treatment of micrognathia in ankyloses of the TMJ in 1999; as a result, mouth opening was improved and facial deformity was corrected. Eski et al (15) reported on three patients with TMJ ankylosis associated with facial asymmetry, who were treated with gap arthroplasty and simultaneous distraction. As a result, TMJ ankylosis was released and bone regeneration was achieved (15). This method used for TMJ ankylosis resulted in reduced treatment time and economic 
burden of the patients, whereas it eliminated the requirement for additional surgery. However, the shortcomings of this procedure are that following release of the ankylotic block, the changes in the mandibular position cannot be completely controlled during the distraction period (12). It must be emphasized that the selection of the surgical procedure depends on the specific condition of the patient, including the type of ankylosis, the severity of mandible dysplasia, the airway obstruction, the patient's age and the family's financial status. Further studies with larger sample sizes are required.

Precision device placement is vital to avoid iatrogenic deformities or surgical failure. In the present case, the accurate osteotomy was guaranteed by using a 3-D printed pre-operative model and a surgical cutting guide. Improvements in the appearance and airway obstruction were apparent. However, it has been reported that the distracted mandible exhibits a period of relapse and usually does not exhibit a 'catch-up' growth compared with the residual somatic growth of the face (16). To achieve optimal appearance and respiratory function, mandibular distraction may be combined with other surgical techniques, e.g. instance genoplasty (17). Mandibular distraction may further correct the 3-D skeletal deficiencies in patients with micrognathia deformity and achieve an ideal surgical effect.

In conclusion, TMJ management with simultaneous mandible distraction is an effective method to improve mandibular movement restrictions, airway obstructions and micrognathia. MDO has a versatile role in the treatment process. Patients with childhood onset require early surgical treatments to avoid secondary malformations. However, poor compliance with post-surgical physiotherapy is likely to lead to a limited range of motion following surgery.

\section{Acknowledgements}

Not applicable.

\section{Funding}

The present study was financially supported by a grant from the National Natural Science Foundation of China (grant no. 81070818).

\section{Availability of data and materials}

The datasets used and/or analyzed during the current study are available from the corresponding author on reasonable request

\section{Authors' contributions}

XY collected, analyzed and interpreted the patient data and was a major contributor in writing the manuscript. JW performed the surgical treatment of the patient and drafted the manuscript. SH contacted the patient and organized return visits and analyzed the patient data. RZ was responsible for acquisition of data and drafting and revising the manuscript. All authors read and approved the final manuscript.

\section{Ethics approval and consent to participate}

Not applicable.

\section{Patient consent for publication}

Written informed consent was obtained from the patient's parents for publication of this case report and accompanying data and images.

\section{Competing interests}

The authors declare that they have no competing interests.

\section{References}

1. Elgazzar RF, Abdelhady AI, Saad KA, Elshaal MA, Hussain MM, Abdelal SE and Sadakah AA: Treatment modalities of TMJ ankylosis: Experience in Delta Nile, Egypt. Int J Oral Maxillofac Surg 39: 333-342, 2010.

2. Chidzonga MM: Temporomandibular joint ankylosis: Review of thirty-two cases. Br J Oral Maxillofac Surg 37: 123-126, 1999.

3. Bookman LB, Melton KR, Pan BS, Bender PL, Chini BA, Greenberg JM, Saal HM, Taylor JA and Elluru RG: Neonates with tongue-based airway obstruction: A systematic review. Otolaryngol Head Neck Surg 146: 8-18, 2012.

4. Breik O, Tivey D, Umapathysivam K and Anderson P: Mandibular distraction osteogenesis for the management of upper airway obstruction in children with micrognathia: A systematic review. Int J Oral Maxillofac Surg 45: 769-782, 2016.

5. Karp NS, Thorne CH, McCarthy JG and Sissons HA: Bone lengthening in the craniofacial skeleton. Ann Plast Surg 24: 231-237, 1990.

6. Kaban LB, Bouchard C and Troulis MJ: A protocol for management of temporomandibular joint ankylosis in children. J Oral Maxillofac Surg 67: 1966-1978, 2009.

7. Resnick CM: Temporomandibular joint reconstruction in the growing child. Oral Maxillofac Surg Clin North Am 30: 109-121, 2018.

8. Gupta H, Tandon P, Kumar D, Sinha VP, Gupta S, Mehra H and Singh J: Role of coronoidectomy in increasing mouth opening. Natl J Maxillofac Surg 5: 23-30, 2014.

9. Yu H, Shen G, Zhang S and Wang X: Gap arthroplasty combined with distraction osteogenesis in the treatment of unilateral ankylosis of the temporomandibular joint and micrognathia. Br J Oral Maxillofac Surg 47: 200-204, 2009.

10. López EN and Dogliotti PL: Treatment of temporomandibular joint ankylosis in children: Is it necessary to perform mandibular distraction simultaneously? J Craniofac Surg 15: 879-884; discussion 884-885, 2004.

11. Sadakah AA, Elgazzar RF and Abdelhady AI: Intraoral distraction osteogenesis for the correction of facial deformities following temporomandibular joint ankylosis: A modified technique. Int J Oral Maxillofac Surg 35: 399-406, 2006.

12. Sharma A, Paeng JY, Yamada T and Kwon TG: Simultaneous gap arthroplasty and intraoral distraction and secondary contouring surgery for unilateral temporomandibular joint ankylosis. Maxillofac Plast Reconstr Surg 38: 12, 2016.

13. Chellappa AL, Mehrotra D, Vishwakarma K, Mahajan N and Bhutia DP: Pre-arthroplastic and simultaneous mandibular distraction for correction of facial deformity in temporomandibular joint ankylosis. J Oral Biol Craniofac Res 5: 153-160, 2015.

14. Papageorge MB and Apostolidis C: Simultaneous mandibular distraction and arthroplasty in a patient with temporomandibular joint ankylosis and mandibular hypoplasia. J Oral Maxillofac Surg 57: 328-333, 1999.

15. Eski M, Deveci M, Zor F and Sengezer M: Treatment of temporomandibular joint ankylosis and facial asymmetry with bidirectional transport distraction osteogenesis technique. J Craniofac Surg 19: 732-739, 2008.

16. Peacock ZS, Salcines A, Troulis MJ and Kaban LB: Long-term effects of distraction osteogenesis of the mandible. J Oral Maxillofac Surg 76: 1512-1523, 2018.

17. Yin L, Tang X, Shi L, Yin H and Zhang Z: Mandibular distraction combined with orthognathic techniques for the correction of severe adult mandibular hypoplasia. J Craniofac Surg 25: 1947-1952, 2014. 